\title{
Two-Wave Multi/Demultiplexer on the Base of Gradient Index Multimode Interference Structures Made by $\mathrm{Ag}^{+}-\mathrm{Na}^{+}$Ion Exchange
}

\author{
M. BŁAHUT* AND K. BAZAN \\ Department of Optoelectronics, Silesian University of Technology \\ Krzywoustego 2, 44-100 Gliwice, Poland
}

\begin{abstract}
The paper presents optimizing investigations by the beam propagation method method of two-wave multi/demultiplexer which uses gradient index multimode interference structures made by $\mathrm{Ag}^{+}-\mathrm{Na}^{+}$ion exchange in BK-7 glass. We have discussed the influence of the structure geometry and technological process parameters for selected wavelength combinations within the range of $635 \div 850 \mathrm{~nm}$. We have described operating parameters of the multiplexers - excess losses and contrasts. We have also presented the way of finding approximate MMI section widths and lengths which satisfy the multiplexing condition.
\end{abstract}

PACS numbers: 42.79.Sz, 42.82.Et

\section{Introduction}

When we excite a multimode waveguide, we can observe the effects involving the matching of input field with mode fields of the multimode waveguide, and then the interference of the excited waves. The intermode interference is accompanied by so called self-imaging effects of input field which is exciting the multimode waveguide. As a result of these effects, the input field, which most frequently comes from a single mode waveguide, or from a group of single mode waveguides, is reproduced as direct, mirrored and multiple images. This phenomenon constitutes a basis for the operation of multimode interference (MMI) structures.

The development of the systems of integrated optics making use of MMI and based on step-index optical waveguides, and, in particular, systems based on semiconductor structures, can be observed since early 1990s $[1,2]$. Step-index MMI structures are applied in splitters and couplers technology, Mach-Zehnder interferometers, phase shifters, modulators and switches. The paper [3] presents an interesting construction of a two-wave multi/demultiplexer for the wavelength $1.3 / 1.55 \mu \mathrm{m}$ having very good parameters in step-index technology on the basis of $\mathrm{SiON}$ waveguides.

In the work [4] it has been demonstrated that the self-imaging effects can occur in gradient waveguides produced by ion exchange method. The aim of this work is to investigate the possibility of two-wave multi/demultiplexer construction in the technology of

\footnotetext{
* corresponding author; e-mail: marek.blahut@polsl.pl
}

gradient index MMI structures made by $\mathrm{Ag}^{+}-\mathrm{Na}^{+}$ion exchange in glass for selected wavelength combinations within the range of $635 \div 850 \mathrm{~nm}$ which is the most useful in sensors applications.

\section{Working principle of two-wave multi/demultiplexer}

The scheme of a two-wave multi/demultiplexer is shown in Fig. 1. It consists of a single-mode waveguide for the wavelength $\lambda_{1}$ and $\lambda_{2}$, a multimode interference section which forms the direct image of the input field for the first wavelength and the image mirrored with respect to the axis of symmetry for the second one. At the places where input field images are formed single mode output waveguides are situated. Depending on the excitation direction, the structure can work as a multi- or demultiplexer.

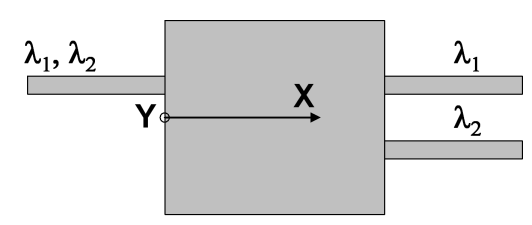

Fig. 1. The scheme of analyzed multi/demultiplexer.

Single-mode waveguides reach the multimode section at $1 / 3$ and $2 / 3$ of their widths. Such an excitation of the MMI section corresponds to restricted interference, a so-called paired interference [1]. Input field images, direct and mirrored ones with respect to the axis of symmetry of the MMI section appear at distances $L_{\mathrm{d}}$, which are 
respectively the even or odd multiplicity of the coupling length $L_{z}$ of the lowest order modes, defined by the equation:

$$
L_{z}=\frac{\pi}{\beta_{0}-\beta_{1}}, \quad L_{\mathrm{d}}=m L_{z}, \quad m=1,2,3, \ldots
$$

where $\beta_{0}$ and $\beta_{1}$ are the propagation constants.

The device length $L_{\mathrm{d}}$ should then satisfy the condition

$$
L_{\mathrm{d}}=m_{1} L_{z, \lambda_{1}}=\left(m_{1}+m_{2}\right) L_{z, \lambda_{2}},
$$

where $L_{z, \lambda}$ is the coupling length for the wave of the wavelength $\lambda, m_{1}$ is an arbitrary integer and $m_{2}$ is an arbitrary odd number.

The waveguide which forms the MMI section is multimode in $X$ direction and single-mode in direction $Y$. This condition restricts the diffusion depth $d=\sqrt{D t_{\mathrm{D}}}$ (where $D$ is self-diffusion coefficient, $t_{\mathrm{D}}$ - time) for the $\mathrm{Ag}^{+}-\mathrm{Na}^{+}$ion exchange process with the maximum refractive index change in BK-7 glass $\Delta n=0.1$. It has to be about $0.3 \mu \mathrm{m}$ for the wavelength $\lambda=0.635 \mu \mathrm{m}$.

\section{Operation range}

The operation range of the demultiplexer results from the modal characteristics of the input waveguide. In Fig. 2 we present the dependences of effective refractive indices of TE modes of the zero and first order on the input waveguide mask widths $w$, obtained by the effective index method. The calculations are performed for the wavelengths equal to $0.635 \mu \mathrm{m}, 0.66 \mu \mathrm{m}, 0.685 \mu \mathrm{m}$, $0.785 \mu \mathrm{m}, 0.808 \mu \mathrm{m}, 0.83 \mu \mathrm{m}, 0.85 \mu \mathrm{m}, 0.98 \mu \mathrm{m}$ which correspond to the most popular laser diodes for visible range and near infrared range [5-7]. Single mode waveguides are produced in $\mathrm{Ag}^{+}-\mathrm{Na}^{+}$ion exchange in $\mathrm{BK}-7$ glass for the diffusion depth of $0.31 \mu \mathrm{m}$.

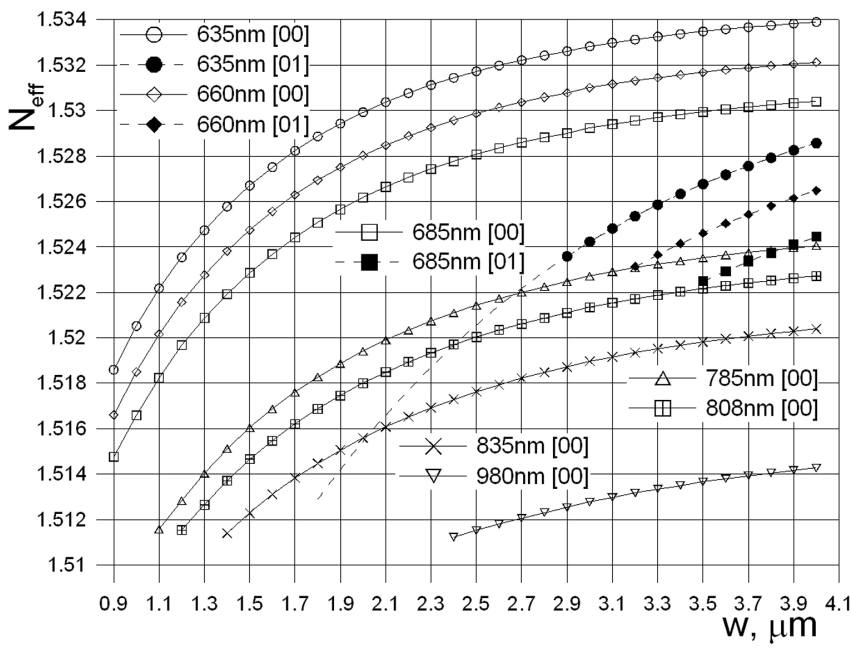

Fig. 2. Effective refractive index $N_{\text {eff }}$ as a function of the mask width $w$ (TE modes). Diffusion depth $d=0.31 \mu \mathrm{m}$

It can be observed basing on the presented characteristic that for the mask width $w$ below $1.5 \mu \mathrm{m}$, corresponding to the cut-off condition of the first order mode of the wavelength $\lambda=0.635 \mu \mathrm{m}$, all modes of the zero order for the wavelength of $0.635-0.835 \mu \mathrm{m}$ are guided. This wavelength interval is the range of examined demultiplexer.

The mask width of the single-mode waveguide equal to $1.3 \mu \mathrm{m}$ has been chosen for further investigations, for which all the zero order modes within the range of the demultiplexer are well guided. In Figs. 3a, b we present field distributions at the output of the single mode waveguide obtained in the diffusion process for the depth $d=0.31 \mu \mathrm{m}$ through the mask opening of the width of $1.3 \mu \mathrm{m}$, for the wavelength of $0.635 \mu \mathrm{m}$ and $0.835 \mu \mathrm{m}$ from the range limits.

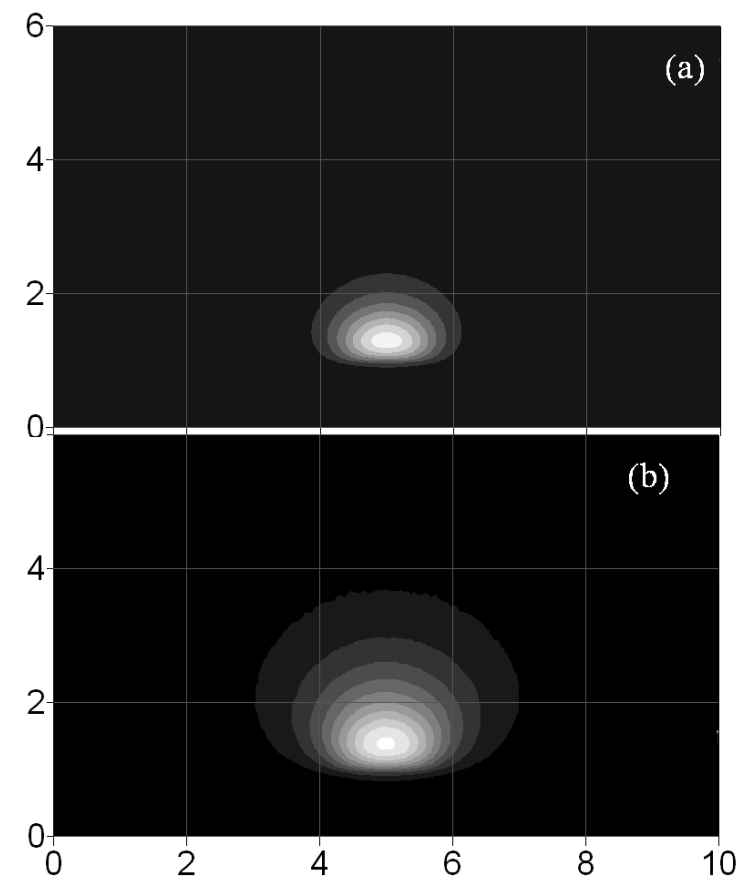

Fig. 3. Numerical simulations by beam propagation method (BPM) method of the field distribution of the fundamental mode in the waveguide obtained in the diffusion process through the mask of the width of $1.3 \mu \mathrm{m}$ for the wavelengths (a) $0.635 \mu \mathrm{m}$, (b) $0.85 \mu \mathrm{m}$.

\section{Multimode interference patterns analysis}

The dependence of propagation length $L_{\mathrm{d}}$ for $N$-fold self-imaging on the multimode waveguide mask width for the ion exchange process is the basic characteristic determining the properties of interference patterns of the MMI section.

In Fig. 4 we present, for example, contour maps of the field distribution along the propagation distance obtained by BPM method for MMI sections excited at $1 / 3$ of the width by the field of the wavelength of $0.635 \mu \mathrm{m}$ for several mask widths $W$.

We can see single images of the input field, direct and mirrored ones, and their multiplicities along the propagation length. Basing on the analysis of such distributions, 


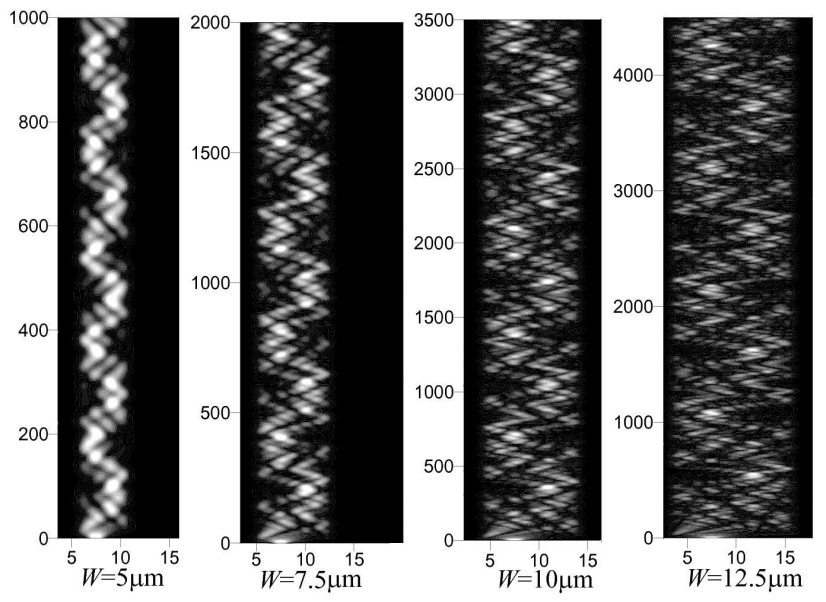

Fig. 4. Contour maps of the field distribution along the propagation distance obtained by BPM method for MMI sections excited at $1 / 3$ of the width by the field of the wavelength of $0.635 \mu \mathrm{m}$.

which have been specified for all wavelengths from the examined range of the demultiplexer and mask widths changing in the interval of $5-15 \mu \mathrm{m}$, the dependence of the propagation length for $\mathrm{m}$-fold single images on the mask width has been determined. In all cases the diffusion depth for the ion exchange process amounts to $0.31 \mu \mathrm{m}$. Figure 5 presents, for example, the characteristic for the wavelength $\lambda=0.830 \mu \mathrm{m}$.

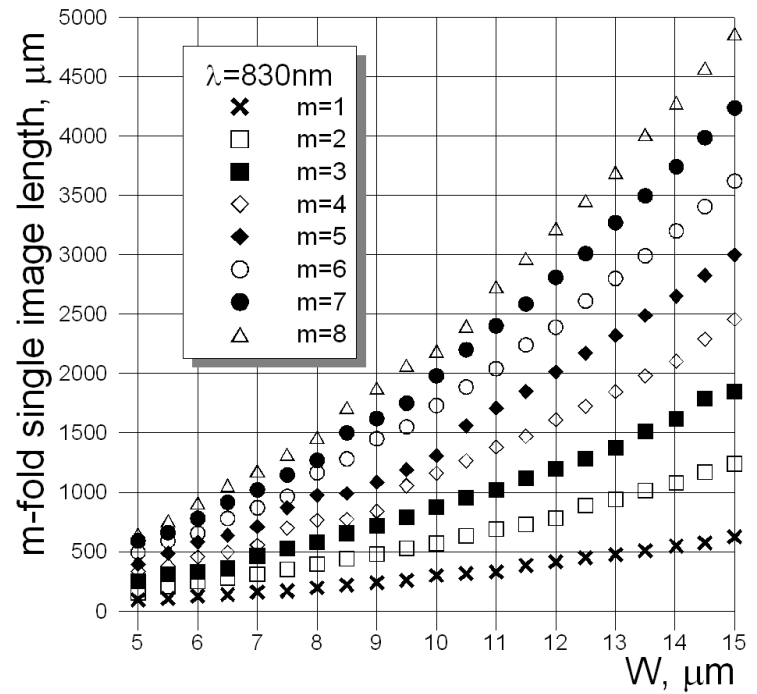

Fig. 5. $\quad M$-fold single image length as a function of a mask width for the wavelength of $0.830 \mu \mathrm{m}$.

\section{Multi/demultiplexer designing}

Comparing the characteristics for different wavelengths it can be observed that there exist MMI section widths for which the mirrored image for the first wavelength and the direct image for the second one appear for the same length of the device. It is illustrated in Fig. 6 for the wavelength $0.635 / 0.830 \mu \mathrm{m}$. As it can be seen for the mask width of $10 \mu \mathrm{m}$ the image mirrored for the wavelength $\lambda_{1}=0.635 \mu \mathrm{m}$ with $m=5$ appears for the same section length as the direct one for the $\lambda_{2}=0.83 \mu \mathrm{m}$ with $m=6$. The characteristic presented in Fig. 7 for the wavelength $0.685 / 0.808 \mu \mathrm{m}$ shows similar relations (for example $W=9 \mathrm{~mm}, m=7$ for $\lambda_{1}=0.685 \mu \mathrm{m}$ and $m=8$ for $\lambda_{1}=0.808 \mu \mathrm{m}$ ). Using analogous dependences the optimal geometries of multi/demultiplexer $0.635,0.66$, $0.685 \mu \mathrm{m} / 0.785,0.808,0.83,0.85 \mu \mathrm{m}$ have been designed.

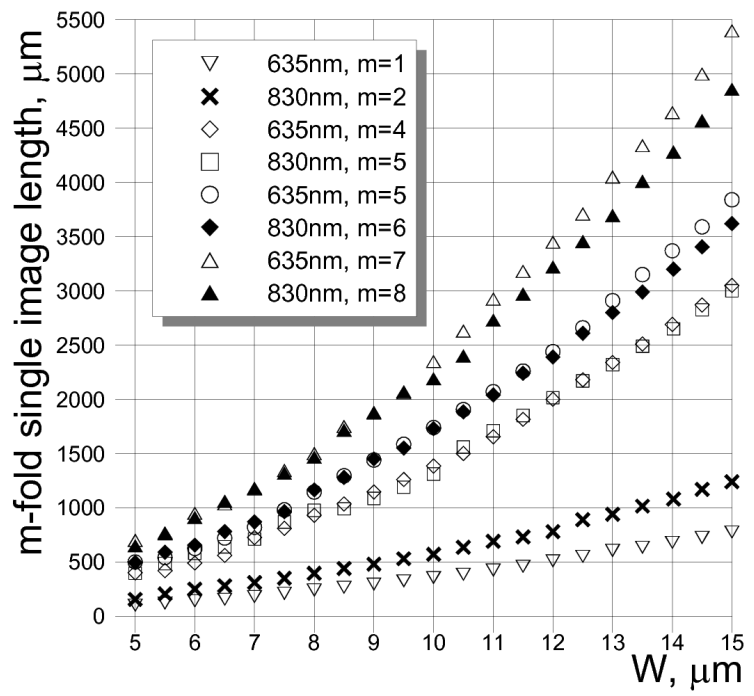

Fig. 6. The comparison of $m$-fold single images lengths for the wavelength $0.635 \mu \mathrm{m}$ and $0.830 \mu \mathrm{m}$ for the different mask widths.

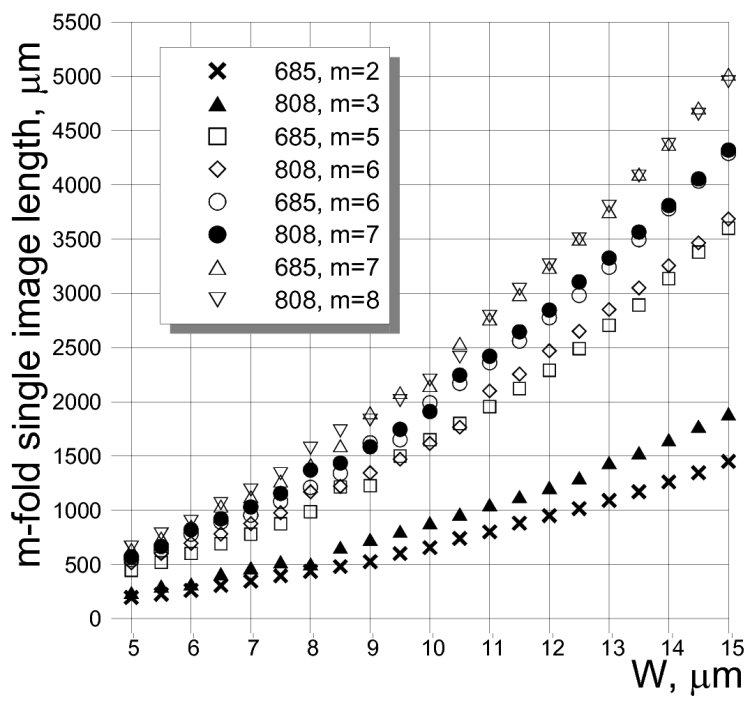

Fig. 7. The comparison of $m$-fold single images lengths for the wavelength $0.685 \mu \mathrm{m}$ and $0.808 \mu \mathrm{m}$ for the different mask widths. 
In Table we have presented the following parameters of the selected two-wave multi/demultiplexer, assuming that the intensities of waves incoming to the structures are $I_{01}=I_{02}=1$ :

- MMI section width $W$ and length $L_{\mathrm{d}}$,

- Intensities in output ports $I_{1}$ and $I_{2}$,

- Contrast $C$ defined as $10 \log \left(I_{1} / I_{2}\right)$ for the wavelength (1) and $10 \log \left(I_{2} / I_{1}\right)$ for the wavelength (2),

- Excess losses $\alpha$ defined as $-10 \log \left(I_{1}+I_{2}\right)$.

Similar values of the parameters have been obtained for other configurations - excess losses which do not exceed $3 \mathrm{~dB}$ and contrast of the order of $20 \mathrm{~dB}$.

TABLE

Multi/demultiplexer parameters.

\begin{tabular}{c|c|c|c|c|c|c}
\hline \hline$L_{\mathrm{d}}[\mu \mathrm{m}]$ & $W[\mu \mathrm{m}]$ & $\lambda[\mu \mathrm{m}]$ & $I_{1}[$ a.u. $]$ & $I_{2}[$ a.u.] & $C[\mathrm{~dB}]$ & $\alpha[\mathrm{dB}]$ \\
\hline 1680 & 9.2 & 0.635 & 0.412 & 0.002 & 23.69 & 3.85 \\
& & 0.785 & 0.021 & 0.449 & 13.29 & 3.48 \\
\hline 2695 & \multirow{2}{*}{14.0} & 0.635 & 0.567 & 0.005 & 20.63 & 2.46 \\
& & 0.808 & 0.003 & 0.602 & 22.68 & 2.20 \\
\hline 1875 & 9.0 & 0.635 & 0.056 & 0.627 & 10.48 & 2.03 \\
& & 0.830 & 0.555 & 0.016 & 15.50 & 2.56 \\
\hline 2720 & \multirow{2}{*}{11.7} & 0.660 & 0.677 & 0.011 & 18.05 & 1.69 \\
& & 0.808 & 0.037 & 0.640 & 12.35 & 1.94 \\
\hline 2775 & \multirow{2}{*}{14.5} & 0.660 & 0.669 & 0.002 & 25.00 & 1.74 \\
& & 0.850 & 0.001 & 0.618 & 28.66 & 2.09 \\
\hline \multirow{2}{*}{3500} & \multirow{2}{*}{12.5} & 0.685 & 0.005 & 0.612 & 20.72 & 2.14 \\
& & 0.808 & 0.543 & 0.014 & 15.77 & 2.65
\end{tabular}

\section{Conclusions}

The paper presents the possibility of two-wave multi/demultiplexer fabrication in the technology of gradient index MMI structures made by $\mathrm{Ag}^{+}-\mathrm{Na}^{+}$ion exchange in glass for wavelength combinations within the range of $635 \div 850 \mathrm{~nm}$. The devices have good optical parameters and can be applied in the optical sensors technology.

\section{References}

[1] L.B. Soldano, E.C.M. Pennings, J. Lightwave Technol. LT-13, 615 (1995).

[2] M. Bachman, P.A. Besse, H. Melchior, Appl. Opt. 33, 3905 (1994).

[3] K.C. Lin, W.Y. Lee, Electron. Lett. 32, 1259 (1996).

[4] M. Błahut, D. Kasprzak, Opt. Appl. 34, 573 (2004).

[5] D. Dorosz, K. Barczak, T. Pustelny, J. Dorosz, Acta Phys. Pol. A 114, A-61 (2008).

[6] T. Pustelny, E. Maciak, Z. Opilski, M. Bednorz, Opt. Appl. 37, 187 (2007).

[7] http://www.roithner. 